\title{
Colistin Use and Its Resistance in Kingdom of Saudi Arabia: A Narrative Review
}

\author{
Hani S. Faidah \\ Department of Medical Microbiology, Faculty of Medicine, Umm Al Qura University, Makkah, Saudi Arabia \\ Email: hsfaidah@uqu.edu.sa
}

How to cite this paper: Faidah, H.S. (2018) Colistin Use and Its Resistance in Kingdom of Saudi Arabia: A Narrative Review. $A d$ vances in Infectious Diseases, 8, 255-261. https://doi.org/10.4236/aid.2018.84021

Received: October 7, 2018

Accepted: November 25, 2018

Published: November 28, 2018

Copyright (C) 2018 by author and Scientific Research Publishing Inc. This work is licensed under the Creative Commons Attribution International License (CC BY 4.0).

http://creativecommons.org/licenses/by/4.0/

\begin{abstract}
Antibiotic resistance is steadily increasing all over the world and has become a major public health challenge. To this end, colistin, an old bactericidal antibiotic of polymyxins family, has been recently re-introduced as only available last-resort antibiotic arsenal for treatment of infections caused by multidrug resistant (MDR)-Gram-negative bacteria. However, the continual and extensive use of colistin has led to the emergence and rapid spreading of its bacterial resistance and non-susceptibility that is currently experiencing a critical healthcare issue with extensive global concern. Both transferable and intrinsic mechanisms of bacterial resistance to colistin have been documented in several countries and, therefore, comprehensive epidemiological data and reports are urgently needed to better understand the current status of this important antibiotic to properly optimize its clinical significance. In consistency, the present narrative review highlights both clinical use and reported bacterial resistance of colistin in the Kingdom of Saudi Arabia.
\end{abstract}

\section{Keywords}

Colistin, Clinical Use, Bacterial Resistance, Kingdom of Saudi Arabia

\section{Introduction}

Polymyxins, a family of cationic polypeptide antibiotics, were discovered in 1947 [1].

Colistin, or polymyxin E, was first isolated in 1949 by the Japanese scientist Koyama from spore-forming soil bacteria and it has been used clinically since 1959 [2] [3] [4]. It was revealed that colistin has a high selectivity against Gram-negative bacteria by binding with their outer lipid a moiety of lipopolysaccharide causing irreversible disruption of their outer membranes with subsequent bactericidal activity [5]. 
Like other polymyxins, the systemic use of colistin, however, was widely discontinued over the past years because of its high rate of nephro- and neurotoxicity. Nevertheless, as the global number of infections caused by MDR-Gram negative bacteria is becoming increased and more prevalent, re-clinical use of colistin, in spite of its toxic effects, has recently get back as the last-resort therapeutic option for treatment of such emergent serious infections [6] [7]. At that regard, colistin has been clinically proved to treat severe infections caused by MDR-Pseudomonas aeruginosa, Klebsiella penumoniae, Acinetobacter baumannii, Haemophilus influenza, Escherichia coli, Salmonella sp, Enterobacter sp, Shigella sp., and other strains of MDR-Gram-negative bacteria [8] [9]. In spite, the extensive use of colistin has led to the emergence of development and spreading of multiple intrinsic and transferable resistant mechanisms and non-susceptibility among the majority of MDR-Gram negative bacterial strains and this critical issue is currently experiencing an extensive concernin several worldwide countries [10]. The present review appraises the clinical use and the reported bacterial resistance of colistin over the recent years in the Kingdom of Saudi Arabia.

\section{Materials and Methods}

A nonsystematic narrative review was performed about the clinical use and bacterial resistance of colistin in the Kingdom of Saudi Arabia. "PubMed" and "EMBASE" were searched using the following keywords: "Colistin" AND "Polymyxins" AND "Saudi Arabia” AND "Use” AND "Resistance" AND "Toxicity". Available publications met to the subject of this review were screened for relevance. Additional data of interest from the Infectious Diseases Society of America (IDSA), and those predicted by WHO, were also included. Publications in non-English language were excluded.

\section{Results and Discussion}

Indeed, there is very little number of valid clinical data covering the different clinical uses of colistin in combating infectious bacterial diseases in Saudi Arabia. However, most of the available data showed that Gram-negative bacteria resistant to the commonly used first line antibiotics are unfortunately continually increasing and colistin seems in most cases as the only available antibiotic medication. With this concept, colistin has been approved in Saudi hospitals for treatment of patient with nosocomial pneumonia caused by MDR - but colistinsensitive-Gram negative bacteria [11]. Constantly, colistin is almost the only attainable therapy for treatment of Saudi patients with pleurisy and ventilator-associated infections caused by carbapenem-resistant Acinetobacter baumannii and Escherichia coli [12]. At that point, addition of intra-pleural colistin therapy along with its conventional intravenous course was found to be more effective [12]. Intrathecal colistin-based therapy was also highly recommended in Saudi Arabia for treatment of critically ill patients with serious nosocomial 
meningitis [13], or post-neurosurgical meningitis due to infection with MDR Acinetobacter baumannii [14]. Favourable synergistic clinical value of combining colistin with ceftolozane-tazobactamhas also been suggested for treatment of infections caused by MDR Pseudomonas aeruginosa [15].

Colistin has been extensively used to treat worldwide infections with MDR-Gram negative bacteria, and this in turn had lead to the development of bacterial resistance and non-susceptibility towards this important. In constancy, a significant number of Saudi studies have been conducted over the past few years to identify the type and rate of colistin-resistance among their patients and to explore their possible underlying mechanisms. For instance, colistin-resistant Enterobacteriaceae spp. and Acinetobacter spp., in particular A. baumannii, have been identified [16] [17], and infections caused by specific strains of Klebsiellapneumoniae with remarkable resistances to colistin have been recently specified [18]. Pathogenic Gram-negative bacteria with well-known intrinsic resistant to colistin such as Proteus, Serratia, and Morganella have widely isolated [16]. More interestingly, MDR Providencia species with dual intrinsic resistance to colistin and carbapenem therapy and causing sever urinary tract infections, e.g., P. stuartii and P. rettgeri; have been recently isolated from hospitalized patients and, exceedingly, such type of resistance could be transmitted to other pathogenic bacteria such as Escherichia coli and vice versa by transformation and conjugation processes [19].

At the molecular level, the plasmid-mediated mobile colistin resistance gene (mcr-1 gene) was successfully detected in isolated resistant bacteria, particularly in samples contained pathogenic Escherichia coli and Klebsiella pneumoniae [10]. In support, mcr-1 gene-mediated bacterial resistance to colistin has been characterized in several worldwide countries [20], and it was detected not only in infected human samples, but also in foods, animals, and environments [21] [22], reflecting its increased horizontal transfer around the world [23]. In addition to the prevalence of $m c r-1$ gene, emergence of Klebsiella pneumoniae harboring mutated $m g r B$-colistin-resistance gene has also been recently raised in Saudi Arabia [18]. Mutations or inactivation in this bacterial gene have previously been identified as the key step in development of bacterial CrrAB and $\mathrm{PhoQ} / \mathrm{PhoP}$ signaling system mediating colistin resistance [24].

In the hospitals and clinical centers, systemic therapy with colistin preparations is generally carried out by intravenous injection. Colistin can also be injected intramuscularly, however, it is not recommended route due to its very painful effect [18]. Most importantly, there is still non optimized systemic dosing strategy regarding the appropriate colistin usage throughout the world [11] [25] [26] and dose-related nephro-and neurotoxicity is an important adverse effect in colistin therapy [27]. Several studies have shown the clinical efficacy of colistin at either low dose of $2.5-5 \mathrm{mg} / \mathrm{kg} /$ day $(1 \mathrm{mg}=12,500 \mathrm{IU})$ or high dose of $5-10 \mathrm{mg} / \mathrm{kg} /$ day regimen of colistin, with variable levels of dose-dependent adverse effects [28]. In Saudi Arabia, the commonly prescribed intravenous colistin doses are between 125,000 IU - 2,500,000 IU per day, which comprises only 
$2.8 \%-41.7 \%$ compared to its proposed optimal dose [13] [29]. Furthermore, the majority of patients were prescribed without an initial loading dose and such practice may not provide adequate early therapy and effective killing [30]. Both sub-optimal dosing and delayed killing practiced have been hypothesized as a part underlying the rising in the number of colistin-resistant bacteria in Saudi Arabia [17] [31]. In support, many worldwide clinical centers have recently considered the foresaid colistin-dosage regimen is sub-optimal, particularly during treatment of carbapenem-resistant Gram-negative bacteria, and they have changed their colistin dosing practice by using a high loading dose in an attempt to optimize its bactericidal efficacy [11] [32] [33]. Nevertheless, application of this modified dosage approach was reported to be associated with high rate of nephrotoxicity in Saudi patients for unknown reason [34]. In addition to that, the introduction of Therapeutic Drug Monitoring (TDM) has shown an improvement in clinical practice of colistin therapy to achieve and maintain its standard average steady-state plasma concentration of $2 \mathrm{mg} / \mathrm{L} \mathrm{[26].} \mathrm{Such}$ benchmark shall standardize colistin dosing across countries at maximum clinical benefits and minimal toxicity. However, routine utilization TDM during colistin therapy is not yet established well in Saudi Arabia [34].

\section{Conclusion}

Like the situation all over the world, colistin has recently been re-clinically used in the Kingdom of Saudi Arabia as last-resort antibiotic to treat and combat serious infections caused by MDR Gram-negative bacteria. However, the emergence of both transferable and intrinsic colistin-resistant bacterial strains is also becoming an important issue. Therefore, colistin resistance control measures, isolation, and characterization of patients infected with colistin-resistant bacteria are an absolute demand. Strict infection control practices are important in Saudi Arabia and other developing countries to repress and hinder the spread of colistin resistance. Any new cases of colistin-resistant bacteria should punctually draw the attention of the infection control sponsors, committees and teams.

\section{Conflicts of Interest}

The author declares no conflicts of interest regarding the publication of this paper.

\section{Funding}

\section{None}

\section{References}

[1] Storm, D.R., Rosenthal, K.S. and Swanson, P.E. (1977) Polymyxin and Related Peptide Antibiotics. Annual Review of Biochemistry, 46, 723-763. https://doi.org/10.1146/annurev.bi.46.070177.003451

[2] Nakajima, S. (1965) Clinical Use of Colimycin Fotic Solution. Jibiinkoka Otolaryngology, 37, 693. 
[3] Ohzawa, R. (1965) The Use of Colimycin Eardrops. Jibiinkoka Otolaryngology, 37, 585.

[4] Falagas, M.E., Kasiakou, S.K. and Saravolatz, L.D. (2005) Colistin: The Revival of Polymyxins for the Management of Multidrug-Resistant Gram-Negative Bacterial Infections. Clinical Infectious Diseases, 40, 1333-1341.

https://doi.org/10.1086/429323

[5] Cai, Y., Chai, D., Wang, R., Liang, B. and Bai, N. (2012) Colistin Resistance of Acinetobacter baumannii: Clinical Reports, Mechanisms and Antimicrobial Strategies. Journal of Antimicrobial Chemotherapy, 67, 1607-1615. https://doi.org/10.1093/jac/dks084

[6] Jeannot, K., Bolard, A. and Plésiat, P. (2017) Resistance to Polymyxins in Gram-Negative Organisms. International Journal of Antimicrobial Agents, 49, 526-535. https://doi.org/10.1016/j.ijantimicag.2016.11.029

[7] Al-Tawfiq, J.A., Laxminarayan, R. and Mendelson, M. (2017) How Should We Respond to the Emergence of Plasmid-Mediated Colistin Resistance in Humans and Animals? International Journal of Infectious Diseases, 54, 77-84.

[8] Li, J., Nation, R.L., Turnidge, J.D., et al. (2006) Colistin: The Re-Emerging Antibiotic for Multidrug Resistant Gram-Negative Bacterial Infection. Lancet, 9, 589-601.

[9] Karvanen, M. (2013) Optimization of Colistin Dosage in the Treatment of Multiresistant Gram-Negative Infections (Dissertation). Uppsala Universiteit, Uppsala.

[10] Leangapichart, T., Gautret, P., Brouqui, P., Mimish, Z., Raoult, D. and Rolain, J.-M. (2016) Acquisition of $m c r-1$ Plasmid-Mediated Colistin Resistance in Escherichia coli and Klebsiella pneumoniae during Hajj 2013 and 2014. Antimicrobial Agents and Chemotherapy, 60, 6998-6999. https://doi.org/10.1128/AAC.01486-16

[11] Cara, A.K.S., Zaidi, S.T.R. and Suleman, F. (2018) Cost-Effectiveness Analysis of Low versus High Dose Colistin in the Treatment of Multi-Drug Resistant Pneumonia in Saudi Arabia. International Journal of Clinical Pharmacy, 40, 1051-1058. https://doi.org/10.1007/s11096-018-0713-x

[12] Rana, M.A., Rahman, B.A., Mady, A.F., Odat, M.A., AlHarthy, A., Ramadan Oel, S., Mumtaz, S.A. and Omrani, A.S. (2014) Intra-Pleural Colistin Methanesulfonate Therapy for Pleural Infection Caused by Carbapenem-Resistant Acinetobacter Baumannii: A Successful Case Report. Infectious Disease Reports, 6, 5413. https://doi.org/10.4081/idr.2014.5413

[13] Bukhary, Z., Mahmood, W., Al-Khani, A. and Al-Abdely, H.M. (2005) Treatment of Nosocomial Meningitis Due to a Multidrug Resistant Acinetobacter baumannii with Intraventricular Colistin. Saudi Medical Journal, 26, 656-658.

[14] Al Shirawi, N., Memish, Z.A., Cherfan, A. and Al Shimemeri, A. (2006) Post-Neurosurgical Meningitis Due to Multidrug-Resistant Acinetobacter baumanii Treated with Intrathecal Colistin: Case Report and Review of the Literature. Journal of Chemotherapy, 18, 554-558. https://doi.org/10.1179/joc.2006.18.5.554

[15] Rico Caballero, V., Almarzoky Abuhussain, S., Kuti, J.L. and Nicolau, D.P. (2018) Efficacy of Human-Simulated Exposures of Ceftolozane-Tazobactam Alone and in Combination with Amikacin or Colistin against Multidrug-Resistant Pseudomonas aeruginosa in an In Vitro Pharmacodynamic Model. Antimicrobial Agents and Chemotherapy, 62, pii: e02384-17. https://doi.org/10.1128/AAC.02384-17

[16] Garbati, M.A., Abdulhak, A.B., Baba, K. and Sakkijha, H. (2013) Infection Due to Colistin-Resistant Enterobacteriaceae in Critically Ill Patients. Journal of Infection in Developing Countries, 7, 713-719. https://doi.org/10.3855/jidc.2851 
[17] Baadani, A.M., Thawadi, S.I., El-Khizzi, N.A. and Omrani, A.S. (2013) Prevalence of Colistin and Tigecycline Resistance in Acinetobacter baumannii Clinical Isolates from 2 Hospitals in Riyadh Region over a 2-Year Period. Saudi Medical Journal, 34, 248-253.

[18] Uz Zaman, T., Albladi, M., Siddique, M.I., Aljohani, S.M. and Balkhy, H.H. (2018) Insertion Element Mediated mgrB Disruption and Presence of ISKpn28 in Colistin-Resistant Klebsiella pneumoniae Isolates from Saudi Arabia. Infection and Drug Resistance, 11, 1183-1187. https://doi.org/10.2147/IDR.S161146

[19] Abdallah, M. and Balshi, A. (2018) First Literature Review of Carbapenem-Resistant Providencia. New Microbes and New Infections, 25, 16-23. https://doi.org/10.1016/j.nmni.2018.05.009

[20] Skov, R.L. and Monnet, D.L. (2016) Plasmid-Mediated Colistin Resistance (mcr-1 Gene): Three Months Later, the Story Unfolds. Eurosurveillance, 21, 30155. https://doi.org/10.2807/1560-7917.ES.2016.21.9.30155

[21] Liu, Y.-Y., Wang, Y., Walsh, T.R., Yi, L.-X., Zhang, R., Spencer, J., et al. (2016) Emergence of Plasmid-Mediated Colistin Resistance Mechanism MCR-1 in Animals and Human Beings in China: A Microbiological and Molecular Biological Study. The Lancet Infectious Diseases, 16, 161-168. https://doi.org/10.1016/S1473-3099(15)00424-7

[22] Baron, S., Hadjadj, L., Rolain, J.M. and Olaitan, A.O. (2016) Molecular Mechanisms of Polymyxin Resistance: Knowns and Unknowns. International Journal of Antimicrobial Agents, 48, 583-591. https://doi.org/10.1016/j.ijantimicag.2016.06.023

[23] Rolain, J.M., Kempf, M., Leangapichart, T., et al. (2016) Plasmid-Mediated MCR-1 Gene in Colistin-Resistant Clinical Isolates of Klebsiella pneumoniae in France and Laos. Antimicrobial Agents and Chemotherapy, 60, 6994-6995. https://doi.org/10.1128/AAC.00960-16

[24] Lippa, A.M. and Goulian, M. (2009) Feedback Inhibition in the PhoQ/PhoP Signaling System by a Membrane Peptide. PLOS Genetics, 5, e1000788. https://doi.org/10.1371/journal.pgen.1000788

[25] Wertheim, H., Van Nguyen, K., Hara, G.L., Gelband, H., Laxminarayan, R., Mouton, J., et al. (2013) Global Survey of Polymyxin Use: A Call for International Guidelines. Journal of Global Antimicrobial Resistance, 1, 131-134. https://doi.org/10.1016/j.jgar.2013.03.012

[26] Nation, R.L., Li, J., Cars, O., Couet, W., Dudley, M.N., Kaye, K.S., et al. (2015) Framework for Optimisation of the Clinical Use of Colistin and Polymyxin B: The Prato Polymyxin Consensus. The Lancet Infectious Diseases, 15, 225-234. https://doi.org/10.1016/S1473-3099(14)70850-3

[27] Spapen, H., Jacobs, R., Van Gorp, V., Troubleyn, J. and Honoré, P.M. (2011) Renal and Neurological Side Effects of Colistin in Critically Ill Patients. Annals of Intensive Care, $1,14$.

[28] Loho, T. and Dharmayanti, A. (2015) Colistin: An Antibiotic and Its Role in Multiresistant Gram-Negative Infections. Acta Medica Indonesiana, 47, 157-168.

[29] Cascio, A., Conti, A., Sinardi, L., Iaria, C., Angileri, F.F. and Stassi, G. (2010) Post-Neurosurgical Multidrug-Resistant Acinetobacter baumannii Meningitis Successfully Treated with Intrathecal Colistin. A New Case and a Systematic Review of the Literature. International Journal of Infectious Diseases, 14, e572-e579. https://doi.org/10.1016/j.ijid.2009.06.032

[30] Markou, N., Markantonis, S.L., Dimitrakis, E., Panidis, D., Boutzouka, E., Karatzas, S., et al. (2008) Colistin Serum Concentrations after Intravenous Administration in 
Critically Ill Patients with Serious Multidrug-Resistant, Gram-Negative Bacilli Infections: A Prospective, Open-Label, Uncontrolled Study. Clinical Therapeutics, 30, 143-151. https://doi.org/10.1016/j.clinthera.2008.01.015

[31] Shibl, A., Al-Agamy, M., Memish, Z., Senok, A., Khader, S.A. and Assiri, A. (2013) The Emergence of OXA-48- and NDM-1-Positive Klebsiella pneumoniae in Riyadh, Saudi Arabia. International Journal of Infectious Diseases, 17, e1130-e1133. https://doi.org/10.1016/j.ijid.2013.06.016

[32] Plachouras, D., Karvanen, M., Friberg, L.E., Papadomichelakis, E., Antoniadou, A., Tsangaris, I., et al. (2009) Population Pharmacokinetic Analysis of Colistin Methanesulfonate and Colistin after Intravenous Administration in Critically Ill Patients with Infections Caused by Gram-Negative Bacteria. Antimicrobial Agents and Chemotherapy, 53, 3430-3436. https://doi.org/10.1128/AAC.01361-08

[33] Mohamed, A.F., Karaiskos, I., Plachouras, D., Karvanen, M., Pontikis, K., Jansson, B., et al. (2012) Application of a Loading Dose of Colistin Methanesulfonate in Critically Ill Patients: Population Pharmacokinetics, Protein Binding, and Prediction of Bacterial Kill. Antimicrobial Agents and Chemotherapy, 56, 4241-4249. https://doi.org/10.1128/AAC.06426-11

[34] Omrani, A.S., Alfahad, W.A., Shoukri, M.M., Baadani, A.M., Aldalbahi, S., Almitwazi, A.A., et al. (2015) High Dose Intravenous Colistin Methanesulfonate Therapy Is Associated with High Rates of Nephrotoxicity: A Prospective Cohort Study from Saudi Arabia. Annals of Clinical Microbiology and Antimicrobials, 14, 3. https://doi.org/10.1186/s12941-015-0062-8 